\title{
Kinetic Transition during Ferrite Growth in Fe-C-Mn Medium
}

\section{Carbon Steel}

C. Capdevila ${ }^{1}$, K. Tanaka ${ }^{2,3}$, J. Cornide ${ }^{1}$, K. Nakanishi ${ }^{2,3}$ and E. Urones-Garrote ${ }^{4}$

${ }^{1}$ Materalia Research Group, Centro Nacional de Investigaciones Metalúrgicas (CENIM-CSIC), Avda. Gregorio del Amo 8, E-28040, Madrid, Spain

2 Toyota Central R\&D Labs., Inc., Nagakute, Aichi 480-1192, Japan

${ }^{3}$ CREST, Japan Science and Technology Agency, Tokyo 100-0004, Japan

${ }^{4}$ Universidad Complutense de Madrid, Centro de Microscopía y Citometría, Av. Complutense s/n, E-28040, Madrid, Spain

\begin{abstract}
The kinetics of austenite-to-ferrite diffusional transformation in a medium carbon Fe-CMn steel was calculated based on classical nucleation and growth theory coupled with CALPHAD multi-component thermodynamics. The description of the growth rate of proeutectoid ferrite includes a time-dependence due to the carbon enrichment in the remaining austenite. The experimental slower kinetics, especially a stagnating behavior at the later stage, has been successfully reproduced when a transition from initial paraequilibrium (PE) to local equilibrium negligible partition (LENP) conditions at austenite:ferrite $(\gamma / \alpha)$ interface was assumed. This transition is allowed when the velocity of moving $\gamma / \alpha$ interface is slow enough to be compared with Mn diffusivity, which leads to built up a Mn spike in the interface. This assumption is consistent with a series of scanning transmission electron microscopy (STEM) analyses for Mn and C
\end{abstract}


which indicates that initial unpartitioned Mn ferrite growth is replaced by partitioned growth.

Keywords: phase transformations, kinetic modeling, medium-carbon manganese steel, forging steel, multicomponent thermodynamics, proeutectoid ferrite.

\section{Introduction}

Medium-carbon manganese steels containing 0.2 0.5 mass percent of $\mathrm{C}$ and $1.0-2.0$ Mn are used in a large quantity as materials for hot forging parts. A prediction of austenite $(\gamma)$ decomposition into a microstructure consisting of proeutectoid ferrite $(\alpha)$, pearlite (P), and bainite (B) has long been a desired technology, which would be the basis for balancing mechanical properties and good machinability of final products. In the quest of an ultimate model that allows predicting accurately the phase fraction produced, an important milestone consists in determining the interface conditions of parent and product phases.

On approaching the subject, one finds out that thermodynamics in Fe-Mn-C ternary system have been well discussed in terms of partitioning of Mn, and the parameters describing diffusion-controlled transformation can now be calculated by CALPHAD method for the ternary and even higher order system. However, there is still discussion on the type of equilibrium operating at the real interface between $\alpha$ and $\gamma,[1]$ although studies devoted to the influence of Mn (among others elements) on the diffusional austenite decomposition reactions in steels has been the subject of academic interest as well as industrial significance for more than a century.[2-9]

In a ternary system in which growth of the ferrite phase is controlled by diffusion it is possible to assume that the phases in contact at the interface are locally in equilibrium 
with each other; this requires that their compositions are given by the ends of a tie-line of the two-phase field in the equilibrium diagram. This can lead to two extremes. At low supersaturation, above the dotted line AB in Fig. 1(a), the interface velocity depends on the diffusion coefficient of $\mathrm{X}$. Below the dotted line $\mathrm{AB}$, the growth velocity depends only on the diffusion coefficient of carbon. In the first case, the carbon concentration gradient in the matrix is negligible, whereas in the second it extends far into the matrix. The $\mathrm{X}$ concentration gradient extends into the matrix in the first case but has only a very sharp and narrow 'spike' in the second case. The first case is usually referred to as local equilibrium partition (LEP) and the second case as local equilibrium no partition (LENP). There is a third condition that represents a constrained equilibrium, in which carbon is in local equilibrium and the alloying element is considered undisturbed by the passage of the interface; this has been termed paraequilibrium (PE). Clearly, no alloying element spike would exist under this second condition. The PE limit is schematized by the $\mathrm{BF}$ line in Fig. 1(a). Figure 1(b) shows the $\mathrm{C}$ and $\mathrm{Mn}$ distribution in $\alpha$ and $\gamma$ according to each local equilibrium condition.

Previous work $[6,10]$ on Mn redistribution on transformation of austenite in Fe-C-Mn alloys has shown two main facts that deserves to be considered. Firstly, that the growth velocity of ferrite is naturally appreciably larger when controlled by carbon diffusion than by substitutional diffusion. Hence, isothermal kinetics of ferrite precipitation divides the ternary $\alpha+\gamma$ two-phase field into two distinct regions: one in which slow ferrite growth is accompanied by $\mathrm{Mn}$ redistribution (partitioning) and one in which faster ferrite growth occurs initially with no long-range redistribution of $\mathrm{Mn}$. The experimental boundary between slow and fast growth regimes has been found to lie between the boundaries predicted by the two limiting cases, i.e. PE and LENP. These limits have been recently discussed in detail elsewhere. [9] Secondly, that the 
impingement of interstitial diffusion fields, for typical austenite grain sizes and reaction temperatures, will often be attained within rather short, and experimentally accessible, reaction times; which is in sharp contrast to the estimated times for substitutional solute field impingement. [11 -12]

Since the growth rate is naturally appreciably larger when controlling by carbon diffusion than substitutional diffusion, many works were focused on differencing both growth mechanisms by indirect measurements such as evolution of ferrite volume fraction and/or ferrite grain sizes with isothermal time. However, a more accurate method to distinguish between two mechanisms is to analyze solute segregation close to interface itself because there is no Mn concentration gradient into the matrix in the case of PE but a very sharp and narrow spike at the interface in the case of LENP occurs. Advanced measurement techniques have cast some light on the grain boundary problem, permitting the evaluation of levels of equilibrium and non-equilibrium segregation. Interphase boundaries, in contrast, have received relatively little attention from experimentalists [13]. It is the purpose of this paper to report results of studies of alloying element $(\mathrm{Mn})$ distributions in the vicinity of (quenched) interfaces of grain boundary nucleated proeutectoid ferrite crystals, formed under substitutional solute partitioning conditions in a Fe-0.37C-1.5Mn steel. Analytical studies of manganese profiles in the vicinity of quenched interfaces linking proeutectoid ferrite and prior austenite have been carried out by means of high resolution microchemical analyses obtained using scanning transmission electron microscopy (STEM).

\section{Materials and Experimental Techniques}

The steel studied (a Fe-0.37C-1.8Mn steel, hereafter named as 37V, with a detailed composition listed elsewhere [14]) was supplied in the form of $50 \mathrm{~mm}$ square bars, 
obtained by conventional casting to a square ingot (2500 kg) and hot rolling to bar. Cylindrical dilatometric test pieces of $3 \mathrm{~mm}$ in diameter and $12 \mathrm{~mm}$ in length were machined parallel to the rolling direction of the bar. The isothermal decomposition of austenite has been analyzed by means of high-resolution dilatometer (Adamel LhomargyDT1000) described elsewhere. [15 -16] The incubation time of allotriomorphic ferrite, or minimum time at which it is possible to find some allotriomorphs nucleated on the austenite grain boundary, is experimentally determined from dilatometric curves obtained during the isothermal decomposition of austenite.

The volume fraction of allotriomorphic ferrite $\left(V_{f}\right)$ was statistically estimated by a systematic manual point counting procedure. [17] A grid superimposed on the microstructure provides, after a suitable number of placements, an unbiased statistical estimation of the $V_{f}$.

A JEOL JEM 3000F transmission electron microscope operating at $300 \mathrm{keV}$ in STEM mode and coupled with an ENFINA 1000 electron energy-loss spectrometer (EELS) has been used in order to determine the evolution of both $\mathrm{C}$ and $\mathrm{Mn}$ through the interface. The spectral data sets were acquired as line-scans normal to the corresponding interface, with an electron probe diameter of $\sim 1.0 \mathrm{~nm}$, a collection time of 12 seconds per EEL spectrum for Mn-L2,3 edge and of 2 seconds for C-K edge, a dispersion of $0.2 \mathrm{eV} / \mathrm{pixel}$ and a collection semi-angle $\beta \sim 15 \mathrm{mrad}$. The profiles of the different elements were extracted by integration of the edge intensity (energy window of $\sim 25 \mathrm{eV}$ ) from each spectrum of the line-scan data set. The background under each individual spectrum was subtracted following a power-law model. [18] The corresponding STEM annular dark field (ADF) images were obtained with a collection semi-angle of 50 mrad.

Specimens were polished in the usual way for metallographic examination. Nital - 2pct etching solution was used to reveal the ferrite microstructure by optical microscopy. 
The specimens for TEM observation were cut to $500 \mu \mathrm{m}$-thick disks from the $3 \mathrm{~mm}$ diameter dilatometric rods, then ground and polished to $50 \mu \mathrm{m}$-thickness. Samples were electropolished with a twin-jet electropolisher at room temperature in a mixture of $5 \%$ perchloric acid, $15 \%$ glycerol and $80 \%$ methanol at $40 \mathrm{~V}$ until perforation occurred. Subsequent thinning in a Gatan precision ion polishing system (PIPS) was performed to reduce the thickness of the observation areas of the samples was performed to about 200 nm.

\section{Results and discussion}

\section{Austenite-to-ferrite transformation}

The isothermal experiments for $37 \mathrm{~V}$ steel were carried out at $973 \mathrm{~K}$. The $\alpha$ nucleated predominantly on austenite grain boundaries and grew into the grains with a morphology that would be described as allotriomorphic (Fig. 2). Toward the end of the reaction, small amounts of intragranular ferrite precipitation were sometimes observed, but these were not found to interfere with the growth kinetic measurements that were taken from the early treatment times. In order to avoid that austenite-to-ferrite transformation was predominantly controlled by nucleation processes instead of growth process, a coarse prior austenite grain size was chosen (a prior austenite grain size with an average diameter $\left(\mathrm{d}_{0}\right)$ of $76 \mu \mathrm{m}$ was achieved after austenitizing temperature of 1523 $\mathrm{K})$. The evolution of the volume fraction of allotriomorphic ferrite formed at $973 \mathrm{~K}$ with isothermal holding time is presented in Fig. 3. The data represented were obtained though standardized quantitative metallography techniques. [19-20] It is clear a decrease in rate of ferrite growth for increasing isothermal times. 


\section{Modeling of austenite-to-ferrite transformation}

Following previous work of Tanaka et al. [21], the austenite-to-ferrite phase transformation could be model as follows. The nucleation rate of $\alpha$ on $\gamma$ boundary surface was calculated following the classical nucleation theory [22]:

$$
I=\frac{K_{1}\left(1-X_{C}^{\gamma}\right) D_{C}^{\gamma}}{T^{1 / 2}} \exp \left(-\frac{K_{2}}{R T \Delta G_{V}^{2}}\right)
$$

where $\Delta \mathrm{G}_{\mathrm{v}}$ is the maximum chemical free energy change per unit volume available for nucleation [23]. The value of $\Delta \mathrm{G}_{\mathrm{V}}$ was determined by CALPHAD method which is a standard tool worldwide for calculating multicomponent thermodynamics. One of the commercial packages, ThermoCalc [24], was introduced in the present modeling work with the help of programming interface, TQ-i. Linking dynamically with ThermoCalc and the steel database TCFe3, driving force $\Delta \mathrm{G}_{\mathrm{V}}$ was calculated for Fe-C- Mn system. On the other hand, the diffusivity of $\mathrm{C}\left(\mathrm{D}_{\mathrm{C}}^{\gamma}\right)$ and $\mathrm{Mn}\left(\mathrm{D}_{\mathrm{Mn}}{ }^{\gamma}\right)$ in austenite were determined with the help of DICTRA software [24]. The parameter $\mathrm{K}_{1}=1.739 \times 10^{26}$ $\mathrm{K}^{0.5} / \mathrm{m}^{4}$ is related to the density of nucleation sites, and $\mathrm{K}_{2}=6.6 \times 10^{8} \mathrm{~J}^{3} / \mathrm{mol}^{3}$ includes the geometry factor of the nuclei as well as the ratio of the interfacial energies for $\gamma / \alpha$ $\left(0.2 \mathrm{~J} \mathrm{~m}^{-2}\right)$ and $\gamma / \gamma\left(0.64 \mathrm{~J} \mathrm{~m}^{-2}\right)$. In this work, we follow the $\mathrm{K}_{1}$ and $\mathrm{K}_{2}$ values reported by Liu et al [25]. As we discuss later, the overall $C$ concentration in austenite $\left(\mathrm{X}_{\mathrm{C}}{ }^{\gamma}\right)$ will change recursively in order to evaluate the soft-impingement. The nucleation, however, saturates soon and the enrichment has no significant effect on I compared with the effect on the growth rate.

After nucleation occurs at the austenite grain boundaries, the thin layers of ferrite that decorate the austenite grain boundaries thicken at a rate controlled mainly by the 
diffusion of $\mathrm{C}$ in the austenite ahead of the advancing $\alpha / \gamma$ interface. The half-thickness of the allotriomorphs is generally calculated under parabolic growth conditions according to the following expression [26]:

$\mathrm{R}=\alpha_{1} \mathrm{t}^{1 / 2}$

where $\alpha_{1}$ is the one - dimensional parabolic growth rate constant and t is the growth time. The value of $\alpha_{1}$ can be obtained by numerical solution from the equation [27]:

$\alpha_{1} \exp \left(\frac{\alpha_{1}{ }^{2}}{4 D_{C}^{\gamma}}\right) \operatorname{erfc}\left(\frac{\alpha_{1}}{2 \sqrt{D_{C}^{\gamma}}}\right)=2\left(\frac{D_{C}^{\gamma}}{\pi}\right)^{1 / 2} \frac{X_{C}^{\gamma}-X^{\gamma \alpha}}{X^{\gamma \alpha}-X^{\alpha \gamma}}$

where $\mathrm{X}_{\mathrm{C}}^{\gamma}$ is the overall carbon content in austenite, $\mathrm{X}^{\gamma \alpha}$ is the austenite solute content at the interface, and $\mathrm{X}^{\alpha \gamma}$ is the ferrite solute content at the interface. ThermoCalc and the steel database TCFe3 were used to determine the interface composition for Fe-C- Mn system. It should be mentioned that the newest version of TQ-i has been upgraded to manage PE constraint. The specific choice of interface carbon composition set for $\mathrm{X}^{\gamma \alpha}$ and $\mathrm{X}^{\alpha \gamma}$ has been matter of some debate, and those in PE constraint would be the most reasonable for an interface moving at higher velocities under high supersaturations. Hillert [9] suggested, however, at a later stage of isothermal transformation, there may be a gradual shift of the interface equilibrium from PE to the one with a spiky partitioning of Mn.

The soft impingement effect (SIE) is pronounced and have to be properly modeled for the $\alpha$ growth of medium carbon steels. De Andrés et al. [14] proposed a 1-D analytical model after Gilmour's work, but in the present model a regressive SIE treatment has 
been implemented to consider $\mathrm{C}$ enrichment caused by a 3-D $\alpha$-shell growing from $\gamma$ grain boundary (see Fig. 4). In equation (4), the $C$ content away from interface $\left(X_{C}{ }^{\gamma}\right)$ should increase if a finite $\gamma$ grain is considered. This could be conveniently monitored by the change in the average $C$ content in remaining $\gamma(\bar{X})$ from the volume fraction of transformed $\alpha\left(\mathrm{V}_{\mathrm{f}}\right)$ as shown in the following equation:

$$
\bar{X}_{C}^{\gamma}=\frac{\bar{X}-V_{f} X_{C}^{\alpha / \gamma}}{1-V_{f}}
$$

The volume fraction of $\alpha\left(\mathrm{V}_{\mathrm{f}}\right)$ was calculated in the following manner [22]. The thickness $\mathrm{R}$ of ferrite nucleating at $\mathrm{T}$ is given by equation (2). Following the Cahn's method [28], ferrite transformation kinetics of grain boundary nucleation was derived in the following manner. The $\alpha$ grains are assumed to present a semielliptical shape having a three times longer axis along the $\gamma / \gamma$ grain boundary than to the center of the grain. Considering a parallel plane at a distance of $y$ from the $\gamma / \alpha$ grain boundary, the extended area of the cross-section ( $\mathrm{Y}^{\mathrm{ex}}$ ) of a growing allotriomorph with the plane is expressed in equation (5). Thus, the $\mathrm{V}_{\mathrm{f}}$ at temperature $\mathrm{T}$ can be calculated by the integral of $\mathrm{y}$ from 0 to the maximum $\mathrm{R}$ value multiplied by the $\gamma$ grain boundary area per unit volume $\left(\mathrm{S}_{\mathrm{V}}\right.$ in equation (6)).

$\mathrm{Y}^{\mathrm{ex}}=\pi \mathrm{I} \Delta \mathrm{t}\left\{9 \mathrm{R}^{2}-\mathrm{y}^{2}\right\}$

$$
\frac{\mathrm{V}_{\mathrm{f}}}{\mathrm{V}_{\mathrm{f}}^{\mathrm{eq}}}=1-\exp \left[-2 \mathrm{~S}_{\mathrm{V}} \int_{0}^{\mathrm{R}}\left\{1-\exp \left(-\mathrm{Y}^{\mathrm{ex}}\right)\right\} \mathrm{dy}\right]
$$


$\mathrm{S}_{\mathrm{V}}$ is related to prior austenite grain size and is estimated to be $2.37 / \mathrm{d}_{0}$ for truncated octahedron austenite grains. A value of $\mathrm{d}_{0}=76 \mu \mathrm{m}$ has been considered. Finally, $\mathrm{V}_{\mathrm{f}}$ must be normalized with respect to the equilibrium fraction $\mathrm{V}_{\mathrm{f}}{ }^{\text {eq }}$ defined by the lever rule.

Figure 5 shows a comparison of calculated ferrite volume fraction formation under PE and LENP as compared with observed results. It is clear that even a soft-impingement effect incorporated through the repetitive calculations of $\mathrm{C}$ enrichment in $\gamma$ has been considered, the observed slow kinetics could not be described with the interface condition under either PE or LENP.

\section{Kinetic transition during ferrite growth}

The boundary between the LEP and LENP regimes of the equilibrium $\gamma+\alpha$ two-phase field and the PE phase boundaries of the Fe-C-Mn system at $973 \mathrm{~K}$ are shown in Fig. 6. Within the region above the LENP line and below the PE phase boundary, the LE treatment predicts very slow a growth accompanied by Mn redistribution and the PE treatment predicts fast ferrite growth unaccompanied by Mn redistribution.

The collection of experimental results shown in Figs. 3 and 5 suggests a transition of interface local equilibrium from PE interfacial conditions at the beginning of the reaction toward LENP conditions at the end of the reaction; otherwise the kinetics should be much faster than the observed if the PE constraint prevails throughout the transformation. The concept of evolving interfacial conditions during transformation has also been suggested by Hillert and his co-workers [1,9].

The treatment here proposed is that the transition from PE to LENP occurs [29]. Two conditions have been considered: Firstly, it is assumed PE interfacial conditions as the starting point, and during growth a Mn spike progressively develops because of an unbalanced flux of Mn across the interface driven by the differences in chemical 
potential of Mn and Fe. Secondly, the efficiency of the cross-interface jumps depends on the interface velocity. The Mn spike will build up more rapidly when the velocity of interface $\left(\mathrm{v}^{\alpha}\right)$ becomes lower compared to Mn diffusivity, since the cross-interfacial jumps will be more efficient.

Consistent with the maximum penetration parameter of substitutional alloying element (i.e. $\mathrm{Mn}$ in the present case study) into $\gamma$ described by Bradley and Aaronson [7], it can be defined the following parameter (p):

$\mathrm{p}=\frac{{ }^{\mathrm{GB}} \mathrm{D}_{\mathrm{Mn}}^{\gamma}}{\mathrm{v}^{\alpha}}=2 \mathrm{n} \frac{{ }^{0} \mathrm{D}_{\mathrm{Mn}}^{\gamma} \sqrt{\mathrm{t}}}{\alpha_{1}}$

where ${ }^{\mathrm{GB}} \mathrm{D}_{\mathrm{Mn}}^{\gamma}$ is the austenite grain boundary diffusion for $\mathrm{Mn}$, which is determined as $n$-times higher than bulk diffusion $\left({ }^{0} \mathrm{D}_{\mathrm{Mn}}^{\gamma}\right)$, and t stands for the growing time. Assuming that Mn cannot diffuse as much as one austenite lattice parameter into $\gamma$ in the reaction time ranges used, it is a sensible approach to consider a value of $\mathrm{p} \sim 10^{-10} \mathrm{~m}$ in accordance to those calculated by Bradley and Aaronson [7]. Therefore, the experimental slow kinetics data presented in Fig. 5, and especially the stagnating behavior at the later stage, has been successfully reproduced assuming a transition from PE to NPLE. Such transition occurs when the $\gamma \rightarrow \alpha$ interface velocity ( ${ }^{\alpha}$ ) is slow enough to allow some Mn diffusion ahead of the interface, or in other words pparameter achieves a certain threshold value. Assuming that the boundary diffusivity of Mn $\left(\mathrm{D}^{\gamma}{ }_{\mathrm{Mn}}\right)$ is several tens of times larger than the bulk diffusivity in $\gamma$, the best fit for experimental results is achieved if a value of $\mathrm{p}=1.6 \times 10^{-10} \mathrm{~m}$ is considered. Comparative overall descriptions of the ferrite growth kinetics with a transition in behavior from PE behavior at short times to LENP behavior together at $\mathrm{p}=1.2 \times 10^{-10}$, 
$1.8 \times 10^{-10}, 2.4 \times 10^{-10}$ are shown in Fig. 7. As discussed previously, a Mn spike progressively develops in reality and the kinetics cannot be assumed to take an abrupt PE to NPLE transition. However, the proposed treatment is a simple approach to predict the kinetics of medium carbon manganese steel, which is of industrial importance in forging parts.

\section{STEM analysis of $\alpha / \gamma$ interface}

The aim of the experimental work to be carried out is to determined if a transition between the constrained equilibrium that represent PE, in which carbon is in local equilibrium and the alloying element (and the solvent element) is considered undisturbed by the passage of the interface; and the LENP condition exists. Since no alloying element spike would exist under PE, the experimental determination of the existence of Mn spike at the interface would clarify is the kinetics transition PE-LENP would exist.

Previous works [30] in the Fe-C-Mn system were focused on the nature (PE or NPLE condition for $\alpha$ growth) but not in the determination of a kinetics transition such is the aim of this work. This transition was theoretically proposed previously by Zurob et al. [31] and experimentally analyzed for Guo et al [32] in Fe-C-Mn-Si by means of STEM analysis, concluding that Mn enrichment was developed locally at the $\gamma / \alpha$ interface for 600s holding at $1024 \mathrm{~K}$. On the other hand, the atom probe tomography analysis carried out by Thullier et al. [30] showed that C/Mn compositions determined were in a certain agreement with the calculated values under the PE constraint. The results reported show the absence of Mn spike in the $\alpha / \gamma$ interface. However, the time analyzed of $50 \mathrm{~s}$ at 973 $\mathrm{K}$ is quite low to allow the build-up of a Mn spike in the interface, i.e. the kinetics 
transition, and they confirm the fact that $\alpha$ follows a PE condition during the early stages of transformation.

In addition, the transition from PE to LENP in low-carbon $(<0.2$ mass\%) steels such as the one studied by Thuillier at al. [30] is difficult to detect through a kinetic change in the volume fraction of proeutectoid ferrite since the difference of $\mathrm{C}$ composition at $\gamma$ side of the $\gamma / \alpha$ interface in PE and LENP were relatively small in terms of the distance from the bulk $\mathrm{C}$ compositions. In medium carbon steels, however, the value of $\bar{X}$ in $\gamma$ comes closer to $\mathrm{X}_{\mathrm{C}}^{\gamma / \alpha}$ so that the shift to LENP interface condition reduces $\mathrm{C}$ diffusion in $\gamma$ and causes a remarkable decrease of $\alpha$ growth kinetics.

The selected condition for STEM analysis is indicated in Fig. 8. They were selected since correspond to a zone were ferrite grow under PE (time of $120 \mathrm{~s}$ ), and the zone were changes in slope were observed (time of 520 s). Figure 9 shows a TEM picture of grain boundary allotriomorphs thickening into austenite grain at both conditions examined.

Figures 10 and 11 show the C-K and Mn-L2,3 edges intensity profiles through $\alpha / \gamma$ interface for the two very different times considered. It is clear that although some partitioning of $\mathrm{Mn}$ is detected after $120 \mathrm{~s}$, this is not substantial and could be due to differences in thickness between ferrite and martensite (it was observed during sample preparation process that ferrite shows higher response to electropolishing than martensite). Figure 12 shows Mn quantification relative to Fe for both case of study and considering thickness differences. The differences in Mn average value between 200 and $600 \mathrm{~s}$ isothermal holding time (120 and $520 \mathrm{~s}$ ferrite growth) could be due to variability in Mn distribution in the matrix, i.e. some Mn banding occurred during processing of the steel analyzed. This was confirmed by EDX analysis in SEM samples. Mn concentration was measured in different samples and the Mn average value varies 
between 1.05 and 1.85 wt.-\%, which is consistent with the STEM results shown in Fig. 13. Figure 13(a) shows that no Mn partition occur between $\alpha$ and $\gamma$ during ferrite growth for isothermal holding times of 200 s (120 s ferrite growth time). Five other line scans have been performed with similar results which allow us to conclude that ferrite grow under PE assumptions at this time range.

An attempt to quantify the $\mathrm{C}$ concentration at both sides of $\alpha / \gamma$ interface has been carried out. However, quantification on EELS implies the relative percentage of elements in the same spectrum [18]. C and (Fe, Mn) are not included in the same spectrum in our experimental data sets in order to optimize the acquisition counts for each edge, and hence it is not possible to supply a value for $\mathrm{C}$ concentration by this method. A tentative comparison between the amounts of $\mathrm{C}$ between both sides of interface is then carried out by areal density data from the spectra. Absolute and quantitative areal density values are extracted if the spectra contain both the core-loss and the low-loss signals. In our case, a C-K edge signal with enough counts cannot be acquired in the same spectrum together with the low-loss data due to saturation of the spectrometer with the extremely intense zero-loss peak. Therefore, although a clear $\mathrm{C}$ partitioning has been observed we have only managed to perform a rough estimation of the ratio of carbon concentration between both sides of $\alpha / \gamma$ interface employing notabsolute areal density values, i.e., values relative to the low-loss region of the spectra. The calculated values of partition coefficient $\left(\mathrm{K}_{\alpha}^{\gamma}\right)$ for $\mathrm{C}$ and $\mathrm{Mn}$ at the $\alpha / \gamma$ interface in austenite under both LENP and PE, and the corresponding measured ones by STEM analysis are listed in Table 1 . The results allow us to conclude that there is a transition from PE to LENP mechanism for proeutectoid ferrite growth in the medium carbon steel studied. Therefore, the stagnation in $\alpha$ growth is a consequence of $\alpha$ growth under LENP conditions. 
Table 1. Partition coefficient $\left(\mathrm{K}_{\alpha}^{\gamma}\right)$ between $\alpha$ and $\gamma$ at $\alpha / \gamma$ interface in austenite

\begin{tabular}{|c|c|c|cc|}
\cline { 2 - 5 } \multicolumn{1}{c|}{} & \multirow{2}{*}{ PE } & \multirow{2}{*}{ LENP } & \multicolumn{2}{c|}{ STEM } \\
\hline $\mathrm{K}_{\alpha}^{\gamma}$ for C & 46.38 & 9.77 & $120 \mathrm{~s}$ & $520 \mathrm{~s}$ \\
$\mathrm{~K}_{\alpha}^{\gamma}$ for $\mathrm{Mn}$ & 1.00 & 4.62 & 1.05 & 4 \\
\hline
\end{tabular}

\section{Conclusions}

1. A model including the time-dependent growth rate and LENP at the $\alpha / \gamma$ interface are intended a Mn-content-sensitive description of the $\gamma \rightarrow \alpha$ transformation kinetics. This has been enabled by the recursive evaluation of $\mathrm{C}$ enrichment in $\gamma$ (soft-impingement effect) and a direct link with CALPHAD thermodynamic calculations.

2. If the PE constraint is imposed consistently at $\gamma / \alpha$ interface, the calculated kinetics is much faster even with the introduction of soft-impingement. The experimental slower kinetics, especially a stagnating behavior at the later stage, has been successfully reproduced when the PE to NPLE transition was allowed to occur. This transition is consistent with previous theoretical works reported $[1,13]$, and it is allowed when the velocity moving $\alpha / \gamma$ interface is slow enough to be compared with Mn diffusivity. This leads to built up a Mn spike in the interface.

3. The transition between PE to LENP regimes has been introduced in the model through the ratio (p) between e Mn diffusion coefficient and the $\alpha / \gamma$ interface velocity. The best fit results from a value of $\mathrm{p}=1.62 \times 10^{-10} \mathrm{~m}$, which is consistent with the values of maximum penetration distances of alloying elements reported by Bradley and Aaronson [7]. 
4. Microanalytical traces across grain boundary ferrite growth fronts in the medium carbon manganese steel transformed at $973 \mathrm{~K}$ are consistent with two proposed growth stages: in the first, rapid, stage, no partition or interfacial accumulation of $\mathrm{Mn}$ is expected; in the second, no partition occurs, but an enrichment of $\mathrm{Mn}$ occurs at the interface.

\section{Acknowledgements}

The authors acknowledge the financial support of Japan Science and Technology Agency (JST). The authors gratefully acknowledge the support of the Spanish Ministry of Science and Innovation for funding this research under the contract MAT2007 63873. J. Cornide acknowledges the Spanish Ministerio de Ciencia e Inovación for financial support in the form of PhD research grant (FPI). C. Capdevila and J. Cornide would like to thanks to Juan Luis Baldonedo from Centro de Microscopía (UCM) for the help in PIPS sample preparation.

\section{References}

[1] H. S. Zurob, C. R. Hutchinson, Y. Bréchet, H. Seyedrezai andG. R. Purdy: Acta Mater., 2009; vol. 57, 2781.

[2] C. Zener: Trans. AIME, 1946, vol. 16, 550.

[3] A. Hultgren: Trans. Am. Soc. Met., 1947, vol. 39, 915.

[4] J. S. Kirkaldy: Can. J. Phys., 1958, vol. 36, 907.

[5] H. I. Aaronson and H. A. Domian: Trans. Metall. Soc. AIME, 1966, vol. 236, 781.

[6] J. B. Gilmour, G. R. Purdy and J. S. Kirkaldy: Metall. Trans., 1972, vol. 3, 3213. 
[7] J. R. Bradley and H. I. Aaronson: Metall. Trans. A, 1981, vol. 12A, 1729.

[8] M. Enomoto and H. I. Aaronson: Metall. Trans. A, 1987, vol. 18A, 1547.

[9] M. Hillert: Scr. Mater., 2002, vol. 46, 447.

[10] C. Capdevila, F. G. Caballero and C. Garcia de Andres: Metall. Mater. Trans. A, 2001, vol. 32A, 661.

[11] C. Capdevila, F. G. Caballero and C. Garcia de Andres: Scr. Mater., 2001, vol. 44, 593.

[12] C. Capdevila, C. Garcia de Andres and F. G. Caballero: Scr. Mater., 2001, vol. 44, 129.

[13] H. Guo and G. R. Purdy: Metall. Mater. Trans. A, 2008, vol. 39, 950.

[14] C. Garcia de Andres, F. G. Caballero and C. Capdevila: Scr. Mater., 1998, vol. 38, 1835.

[15] F. G. Caballero, C. Capdevila and C. Garcia de Andres: Mater. Sci. Technol. 2001, vol. $17,1114$.

[16] Carlos Garcia de Andres, F. G. Caballero, C. Capdevila and D. San Martin: Mater. Charact., 2002, vol. 49, 121.

[17] F. G. Caballero, C. Capdevila and C. Garcia de Andres: Scr. Mater., 2000, vol. 42, 1159.

[18] R. F. Egerton:. Electron energy-loss spectroscopy in the electron microscope, Plenum Press, New York, 1996.

[19] C. Capdevila, F. G. Caballero and C. Garcia de Andres: Metall. Mater. Trans. A, 2001, vol. 32A, 661.

[20] C. Capdevila, F. G: Caballero and C. Garcia de Andres: ISIJ Int., 2001, vol. 41, 1083. 
[21] K. Tanaka, Y. Yogo, K. Nakanishi and C. Capdevila: Mater. Trans., 2009, vol. 50, 551.

[22] M. Umemoto, A. Hiramatsu, A. Moriya, T. Watanabe, S. Nanba, N. Nakajima, G. Anan, Y. Higo: ISIJ Int., 1992, vol. 32, 306.

[23] M. Hillert, Lectures in the theory of phase transformations, H.I. Aaronson (ed), TMS-AIME, Warrendale, PA, 1975, 1.

[24] Thermo-Calc Software, Stockholm Technology Park, Stockholm, Sweden, http://www.thermocalc.com/.

[25] X. Liu, P. Kayjalainen, J. S. Perttula: Proc. 2nd Int. Conf. on Modeling of Metal Rolling Processes. Plenum Press, London, 1996.

[26] C. Zener: J. Appl. Phys., 1949, vol. 20, 950.

[27] J. W. Christian: Theory of Tranformations in metals and alloys Part I 3rd ed, Pergamon, Oxford, 2002, 808.

[28] J.W. Cahn: Acta Metall., 1956, vol. 4, 449.

[29] K. Tanaka, M. Hara, Y. Yogo, K. Nakanishi and C. Capdevila:. Current Advance in Materials Processing of Iron and Steel Institute of Japan (CAMP-ISIJ), 2008, vol. 21, 469.

[30] O. Thuillier, F. Danoix, M. Gouné and D. Blavette: Scr. Mater., 2006, vol. 55, 1071.

[31] H. S. Zurob, C. R. Hutchinson, A. Béché, G. R. Purdy and Y. Bréchet: Acta Mater., 2008, 56, 2203.

[32] H. Guo, G. R. Purdy, M. Enomoto and H. I. Aaronson: Metall. Mater. Trans. A, 2006, vol. 37, 1721. 


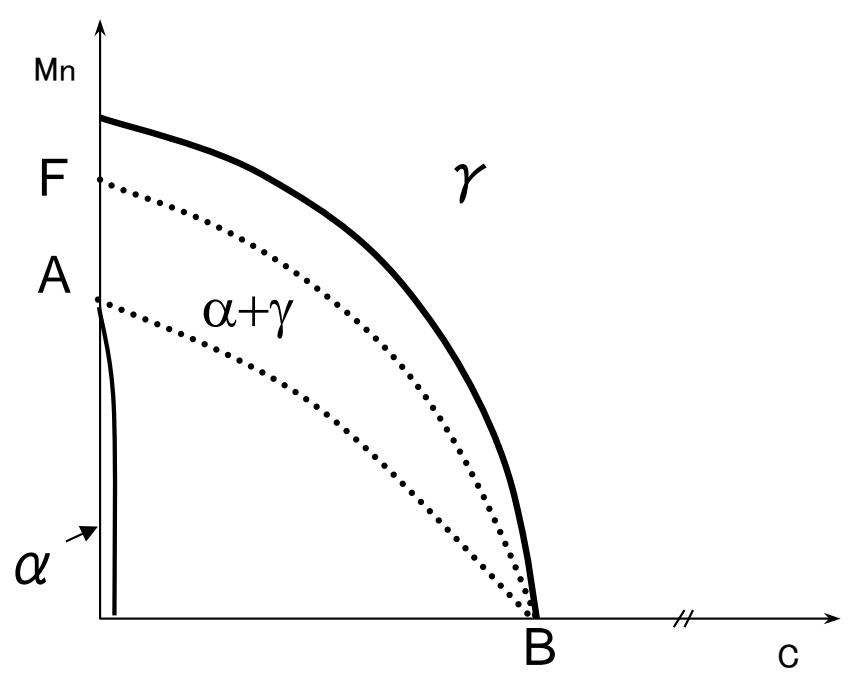

(a)

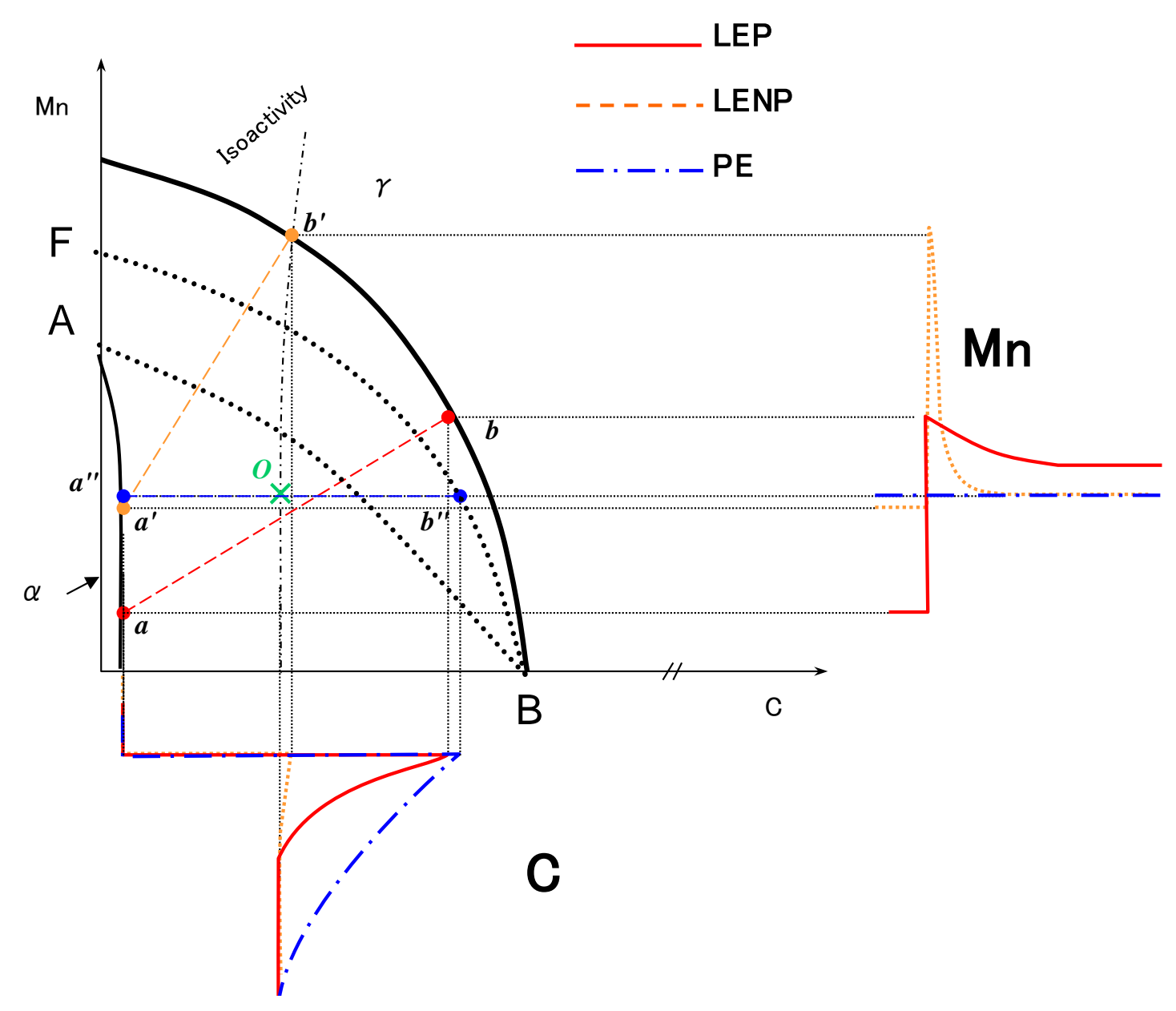

(b)

Figure 1. Schematic representation for (a) LEP (solid line), LENP (dotted $\mathrm{AB}$ line) and $\mathrm{PE}$ (dotted FB line) conditions and (b) $\mathrm{C}$ and $\mathrm{Mn}$ profiles in $\alpha$ and $\gamma$ in a Fe-C-Mn system 


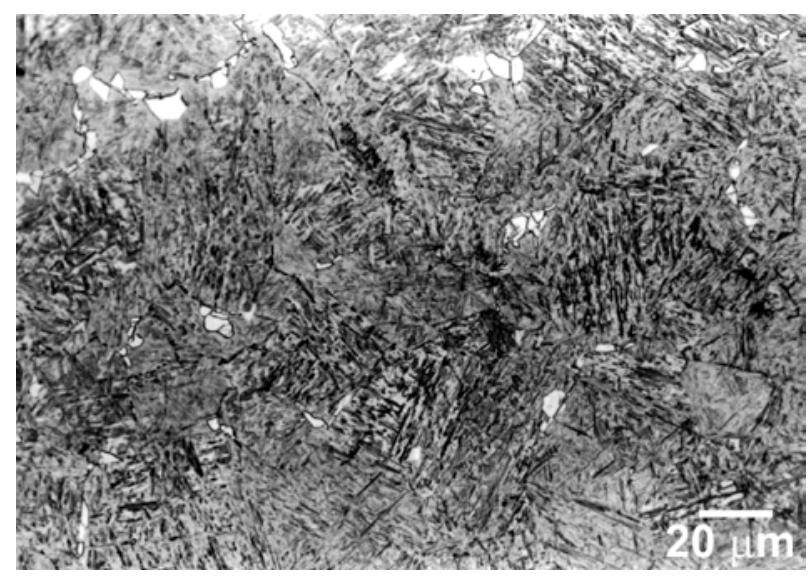

(a)

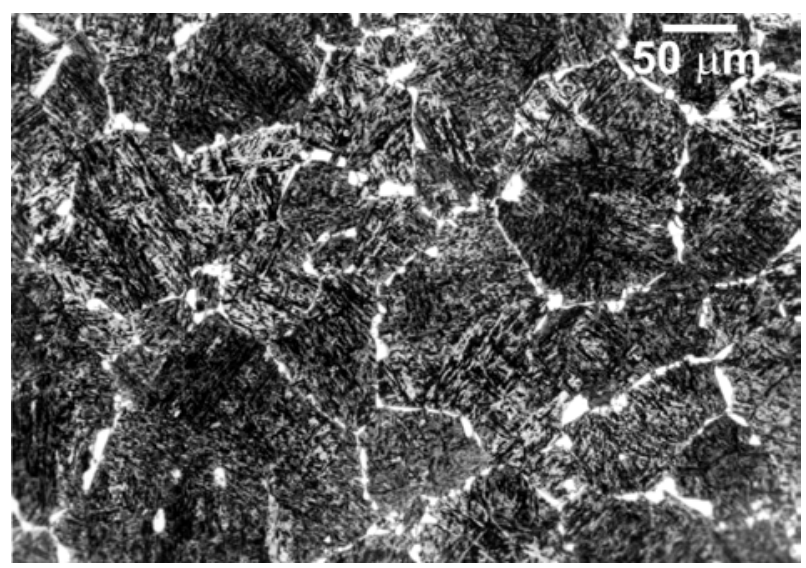

(b)

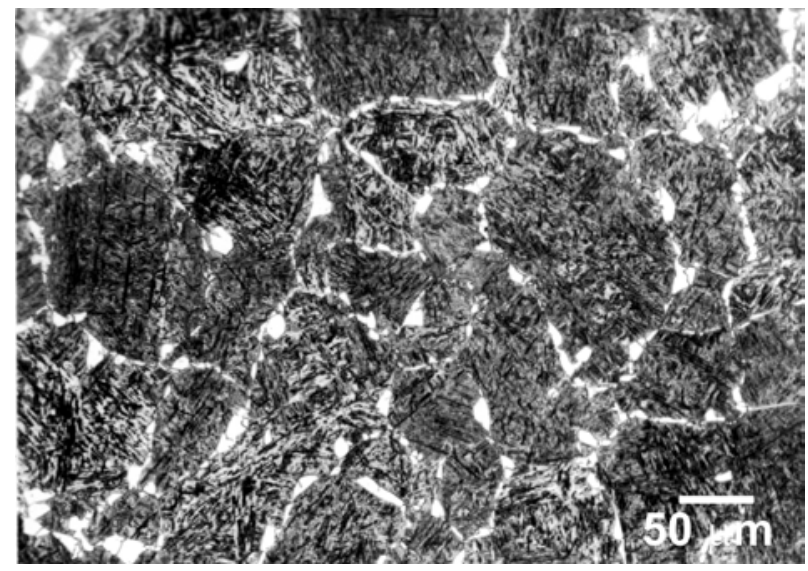

(c)

Figure 2. Microstructures obtained after isothermal holding at $973 \mathrm{~K}$ for (a) $200 \mathrm{~s}$, (b) $700 \mathrm{~s}$ and (c) $1200 \mathrm{~s}$ 


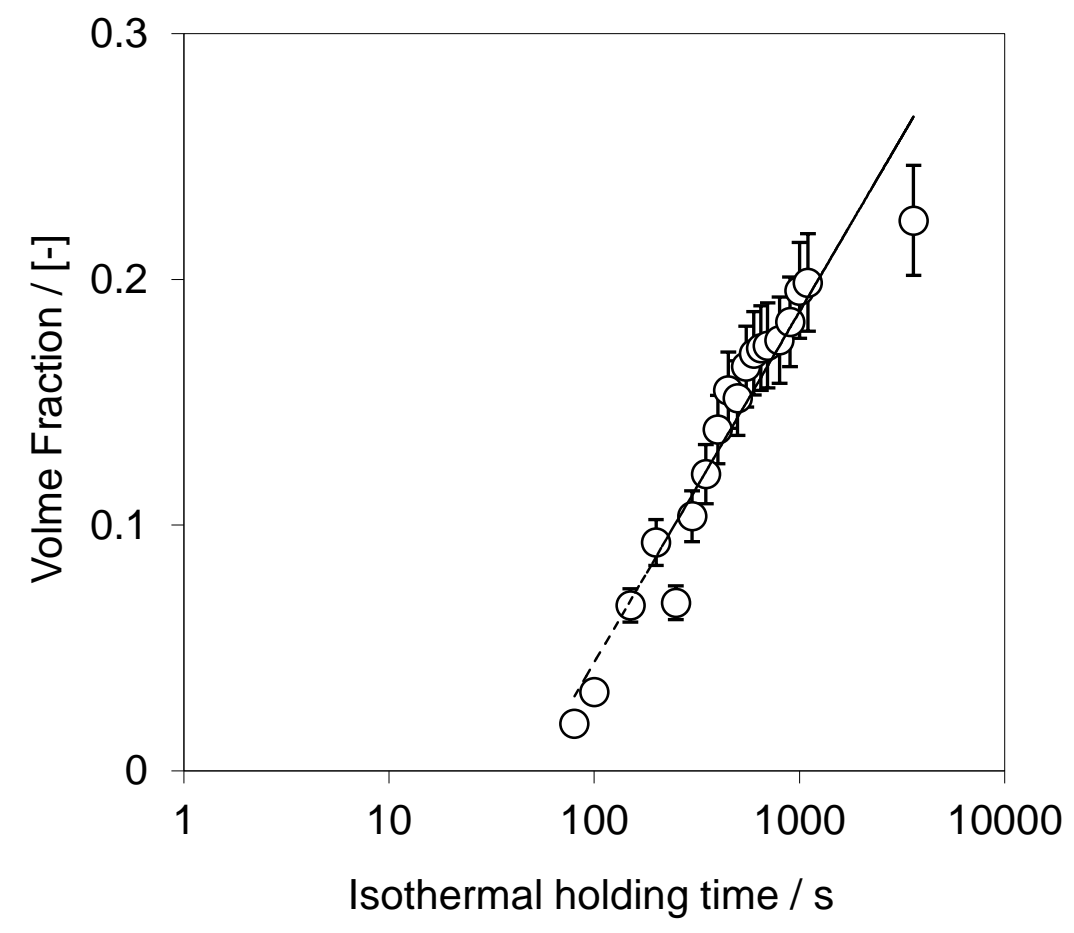

Figure 3. Evolution of volume fraction of allotriomorphic ferrite with isothermal holding time during holding at $973 \mathrm{~K}$ 


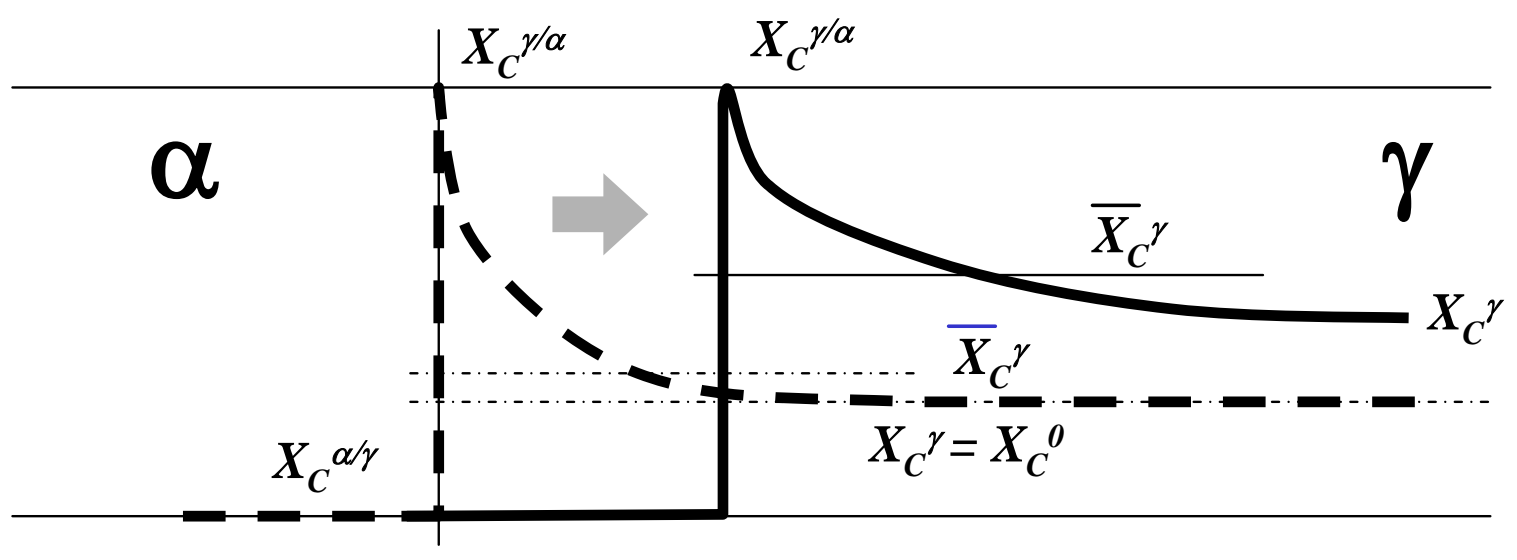

Figure 4. Scheme to illustrate the SIE in medium carbon steels. Dashed lines stand for initial state and solid lines for growing. 


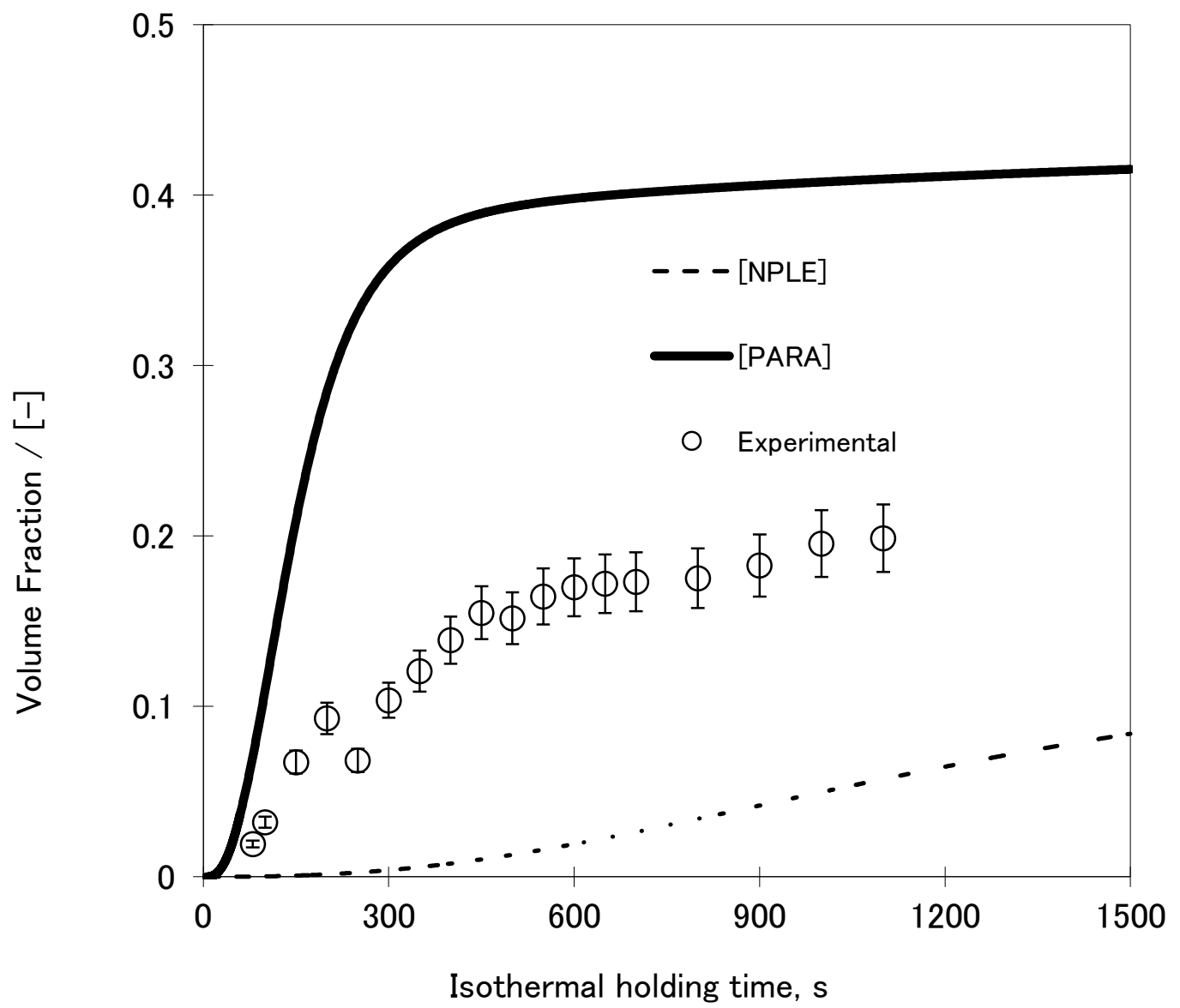

Figure 5. Comparison between experimental and calculated values of ferrite volume fraction under PE and NPLE conditions. 


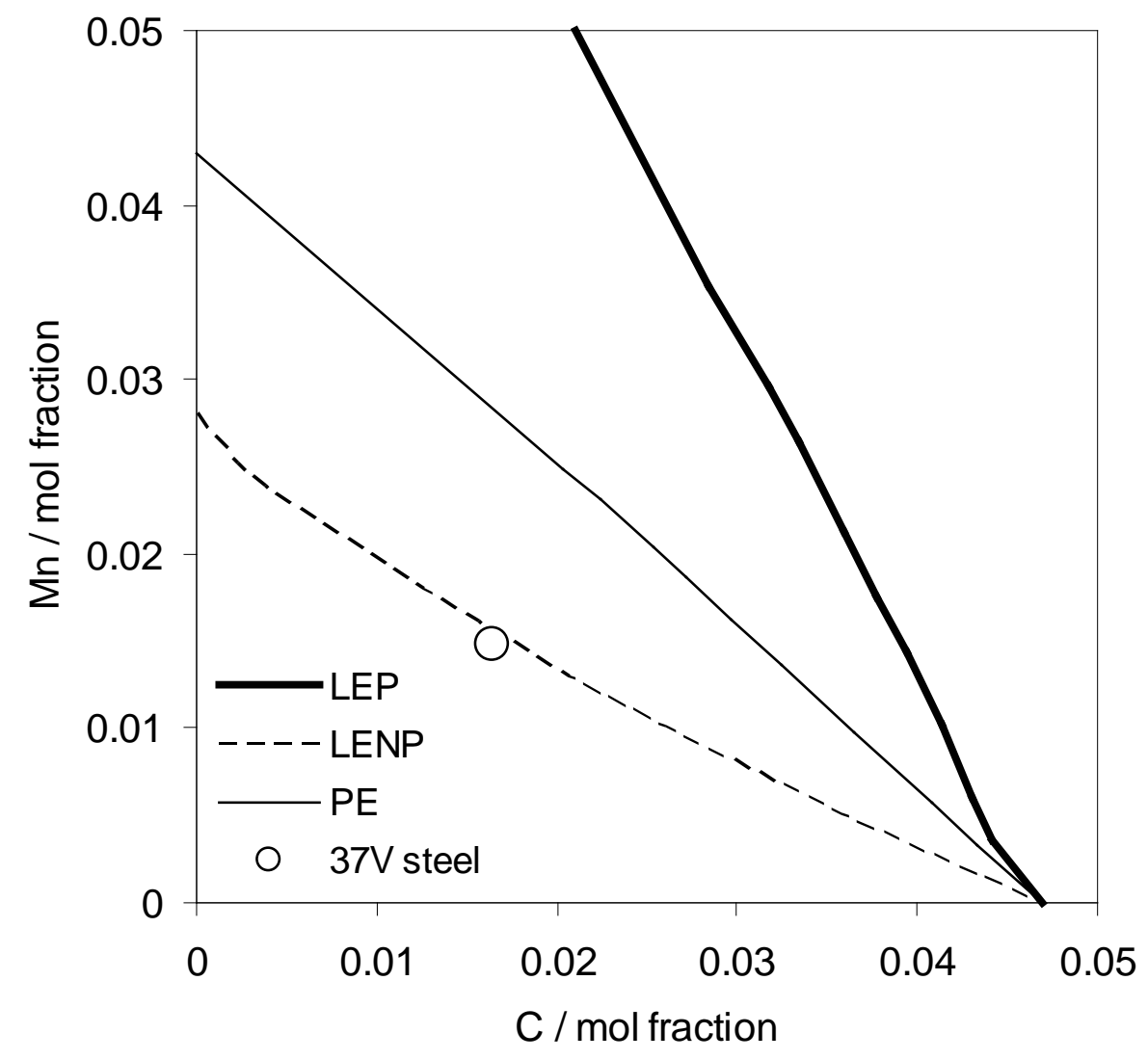

Figure 6. Isothermal section of the Fe-C-Mn ternary phase diagram at $973 \mathrm{~K}$. The bold lines represent equilibrium phase boundaries, the dashed line is the LEP/LENP boundary, and the fine solid lines are the PE phase boundaries. The steel composition examined is indicated by the open circle. 


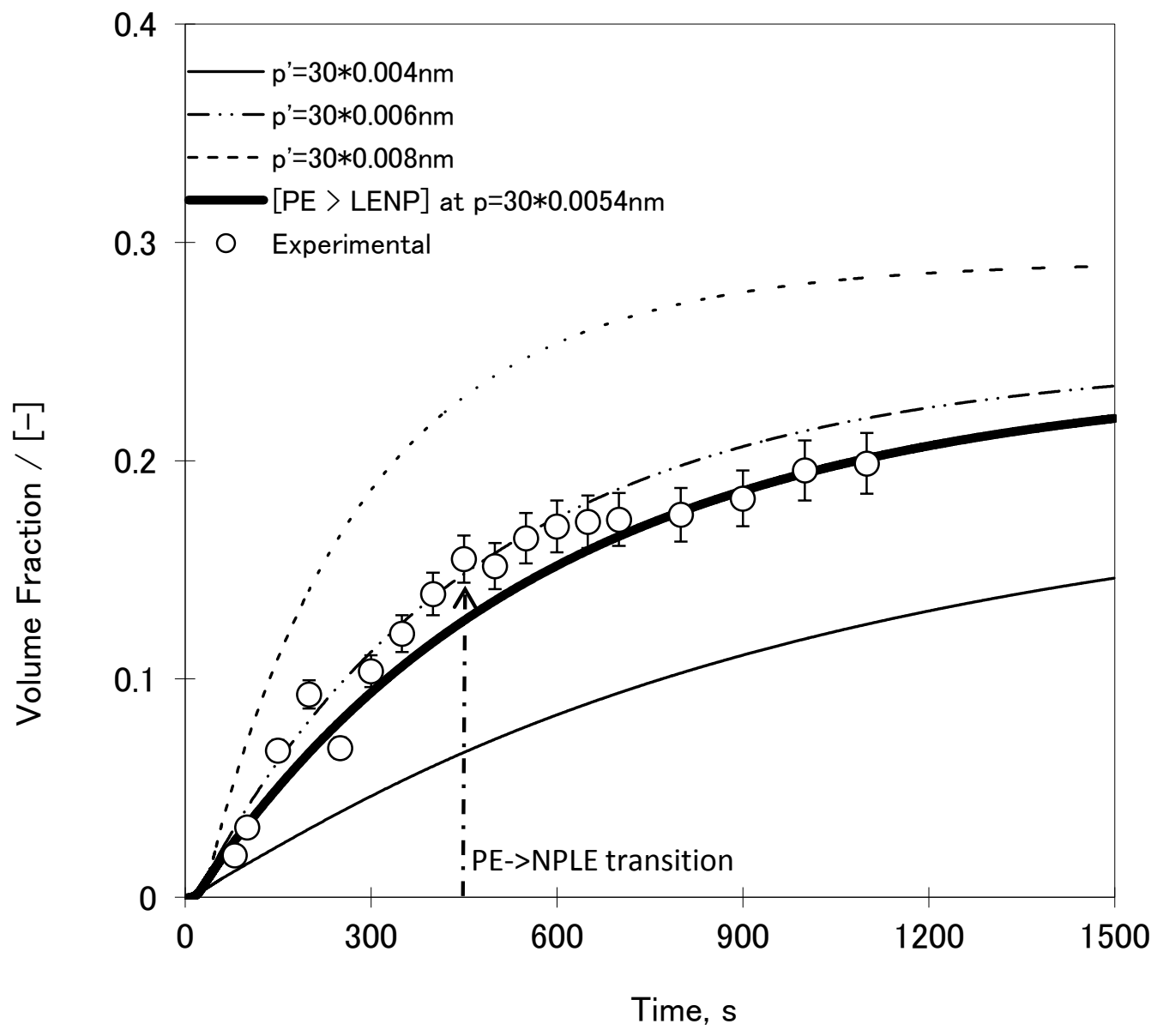

Figure 7. Postulated transition PE $\rightarrow$ NPLE in basis of good fitting to experimental dataset. 


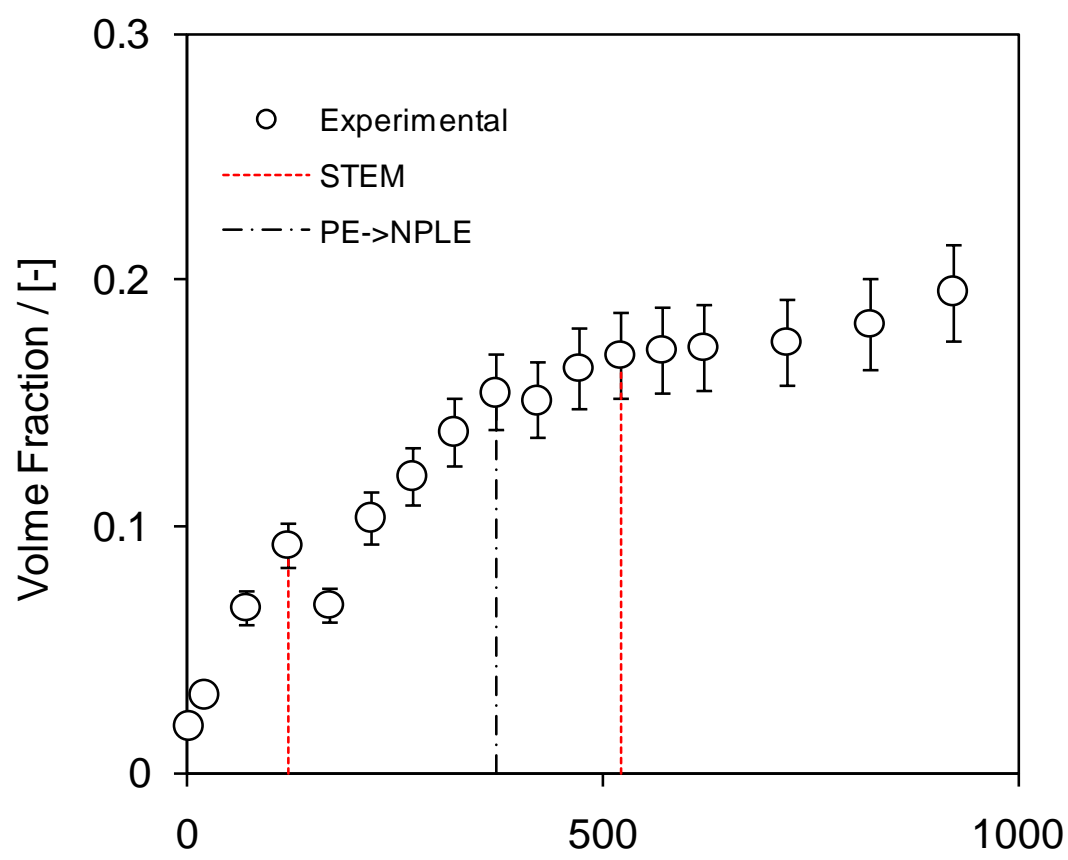

Ferrite growth time / $\mathrm{s}$

Figure 8. Conditions for STEM analysis of $\alpha / \gamma$ interface. 


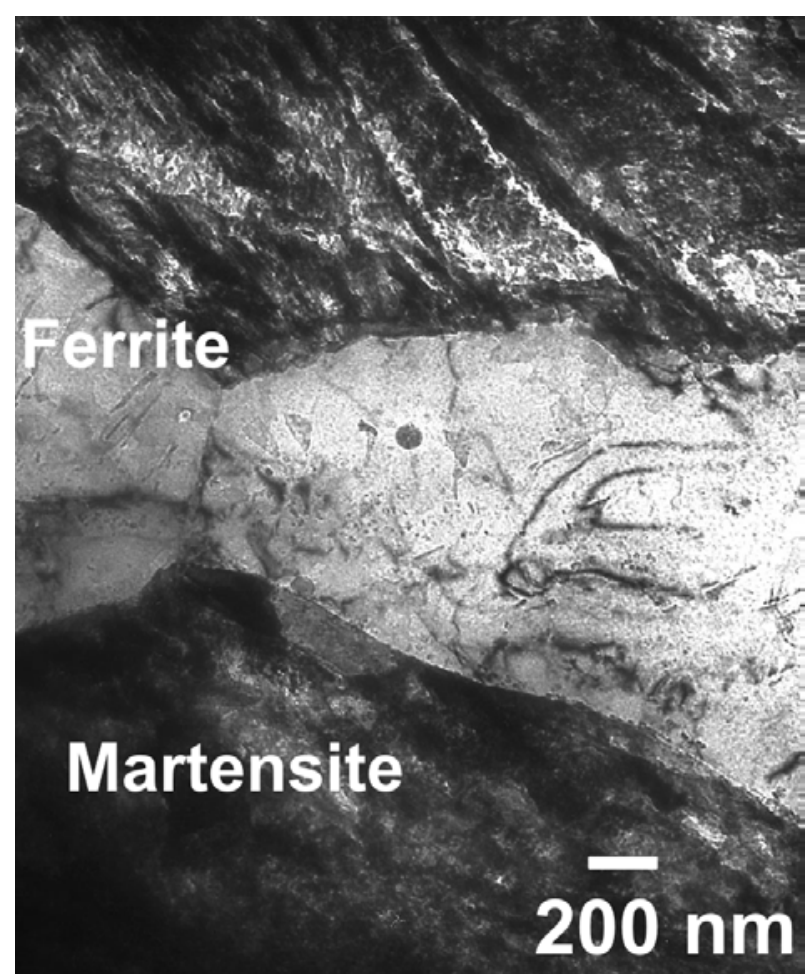

(a)

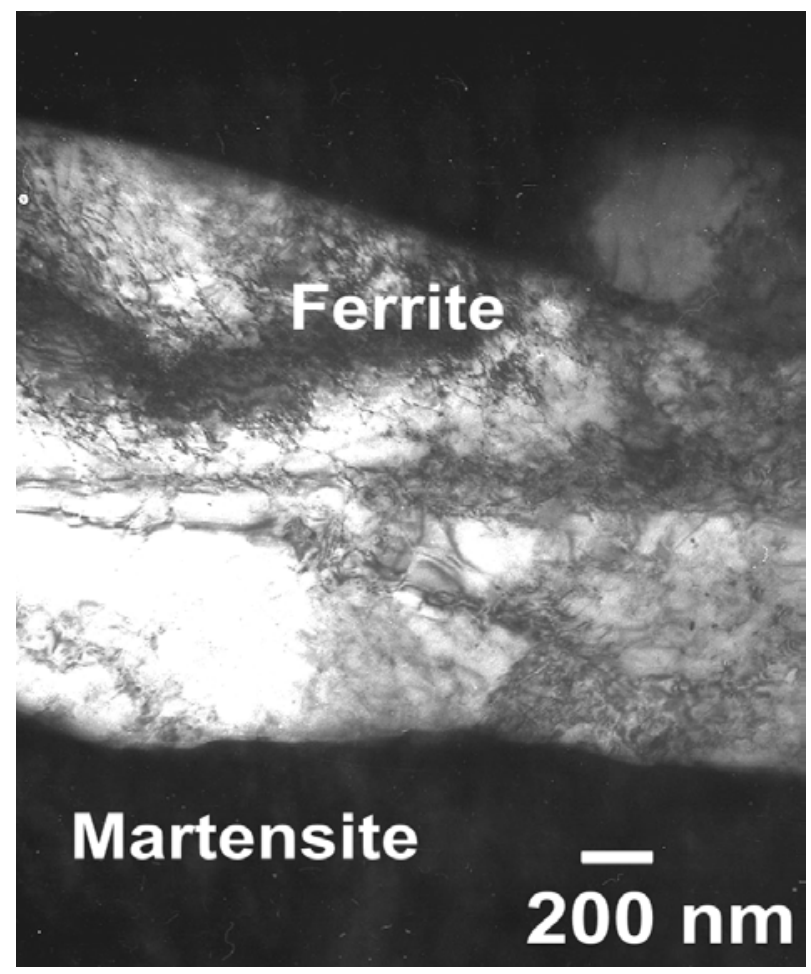

(b)

Figure 9. TEM micrograph of allotriomorphic ferrite formed at $973 \mathrm{~K}$ after (a) $200 \mathrm{~s}$ and (b) $600 \mathrm{~s}$ holding time. 

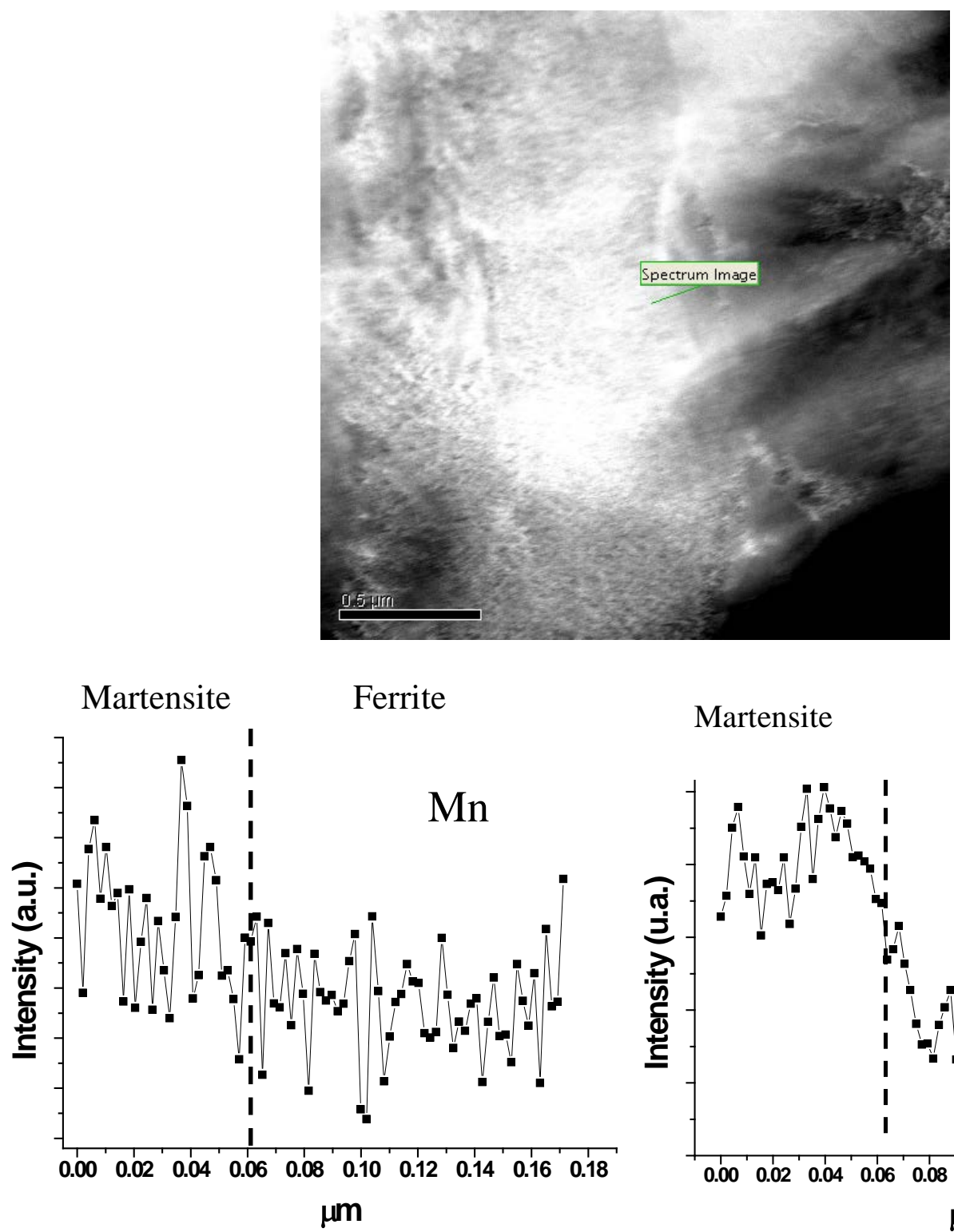

Martensite

Ferrite

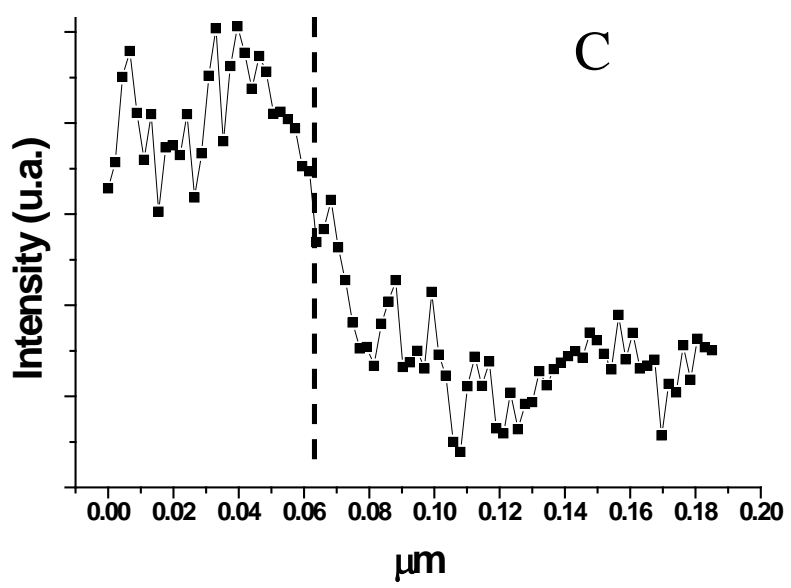

Figure 10. $\mathrm{Mn}$ and $\mathrm{C}$ profiles across the $\alpha / \gamma$ interface after $120 \mathrm{~s}$ of ferrite growth time. 


\section{Martensite}

Ferrite
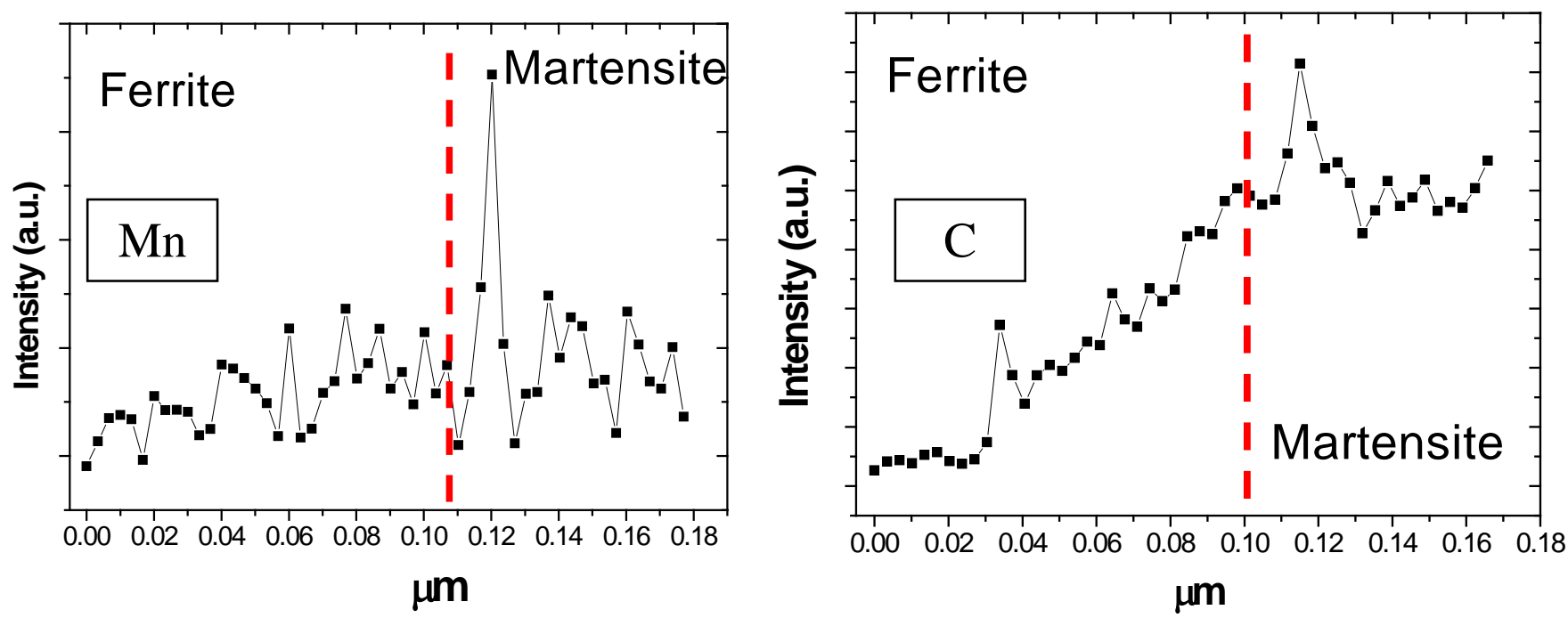

Figure 11. Mn and C profiles across the $\alpha / \gamma$ interface after $520 \mathrm{~s}$ of ferrite growth time. 


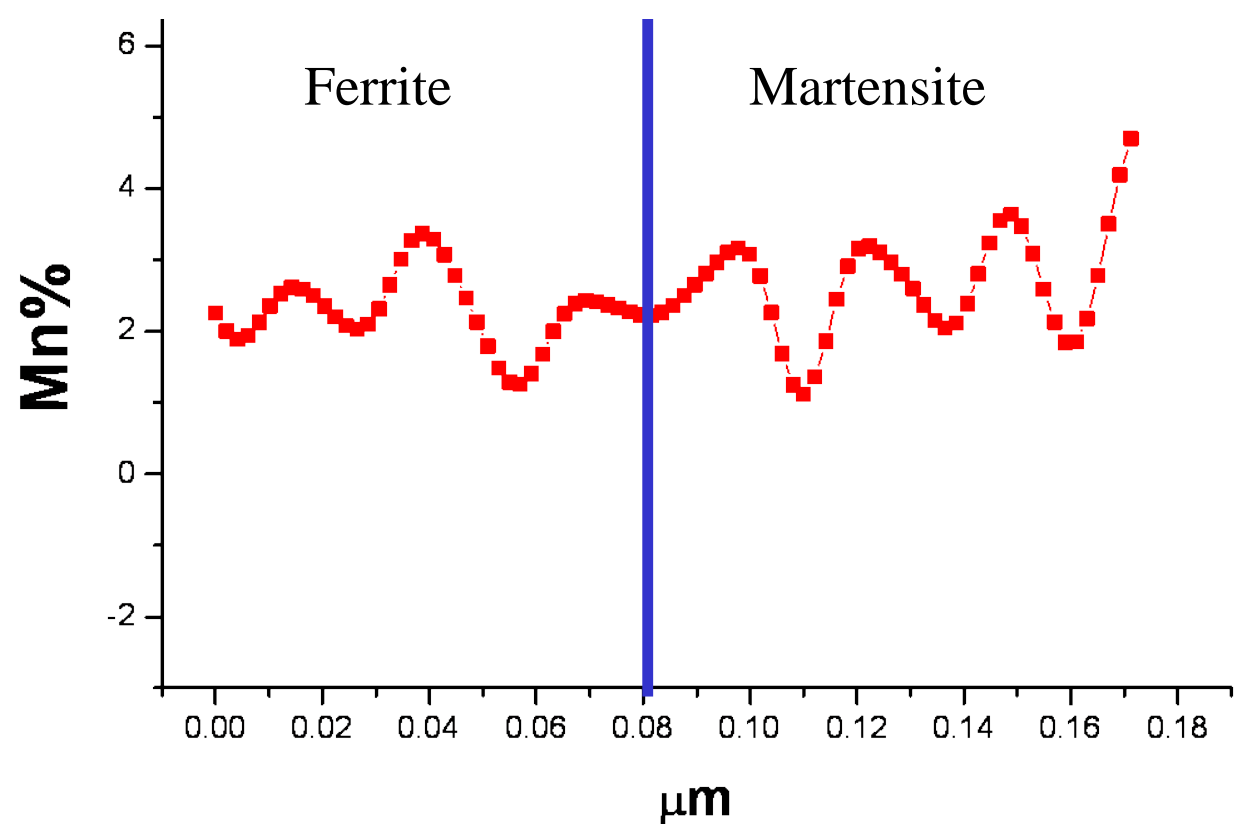

(a)

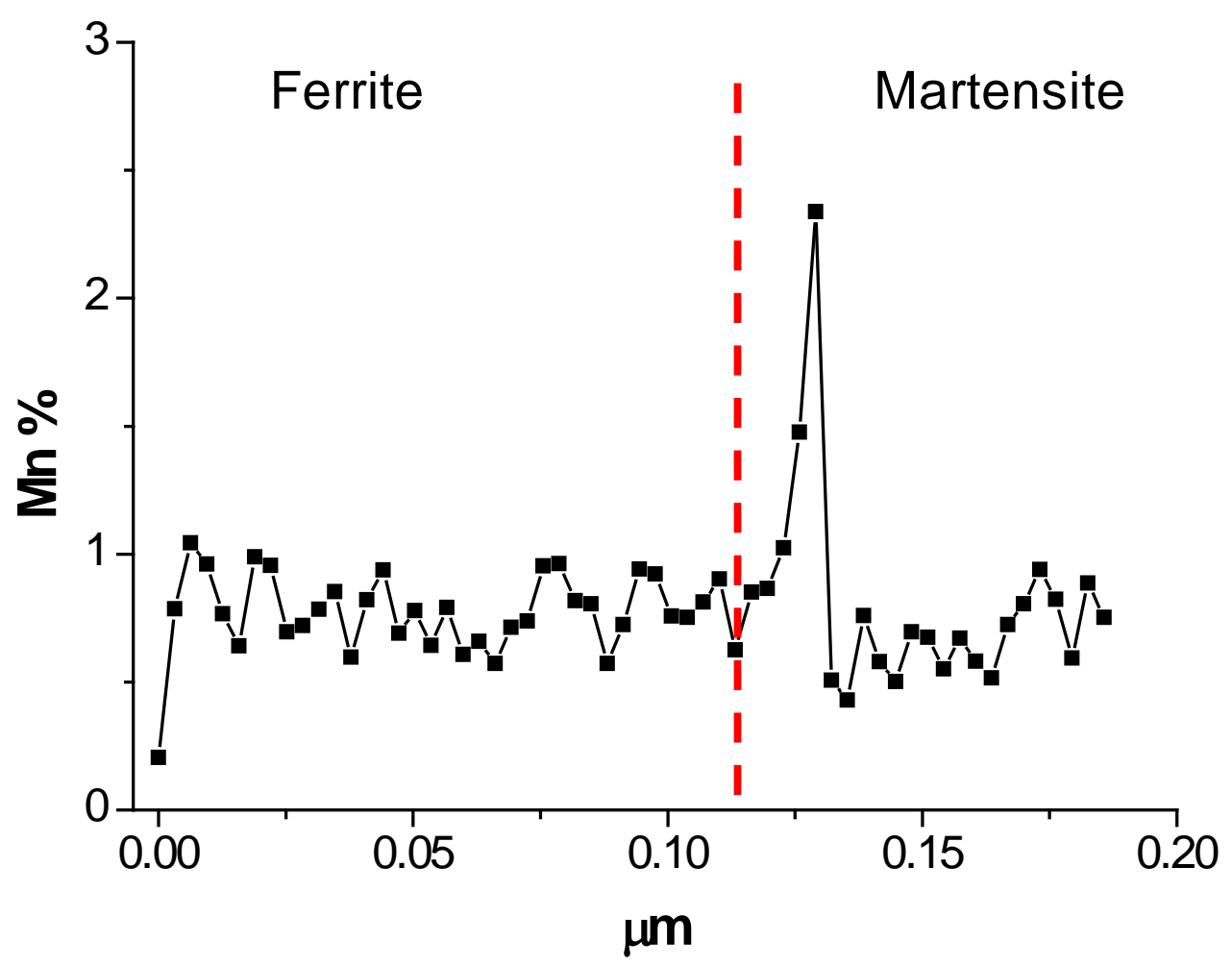

(b)

Figure 12. Mn quantification after (a) 120s and (b) 520 s of ferrite growth time. 